Series A

I. MATHEMATICA

322

\title{
ON THE NULL-SETS FOR EXTREMAL DISTANCES
}

\author{
B. \\ JUSSI VÄISÄL $\ddot{A}$
}

HEL S I N I 1962

S U O M A A I N E T IEDEAKATEM I A

https://doi.org/10.5186/aasfm.1963.322 
Communicated 14 September 1962 by F. Nevanlinna and O. Lehto. 


\section{On the null-sets for extremal distances}

1. Introduction. Let $\bar{R}^{n}$ be the $n$-dimensional Möbius space, i.e. the one point compactification of the Euclidean $n$-space $R^{n}$. Let $E$ be a closed proper subset of $\bar{R}^{n}$. Suppose that $F_{1}$ and $F_{2}$ are two disjoint continua in the complement of $E$. Denote by $\bar{T}$ the family of all arcs in $\bar{R}^{n}$ which join $F_{1}$ and $F_{2}$ and let $\Gamma_{E}$ be the subfamily of $\Gamma$ whose members do not meet $E$. Consider the modules $\left.{ }^{1}\right) M(\Gamma)$ and $M\left(\Gamma_{E}\right)$. Since $\Gamma_{E} \subset \Gamma$, $M\left(\Gamma_{E}\right) \leqq M(\Gamma)$.

We say that $E$ is a null-set for extremal distances (with respect to $\bar{R}^{n}$ ) if $M\left(\Gamma_{E}\right)==M(T)$ for all pairs of sets $F_{1}, F_{2}$. In other words, the removal of $E$ does not change the extremal distance between two continua in its complement. We abbreviate this by saying that $E$ is an NED-set or simply $E$ is NED.

In the case $n=2$, Ahlfors and Beurling [1] have proved that $E$ is NED if and only if it is an $O_{A D}$-set, i.e. every non-constant analytic function in the complement of $E$ has an infinite Dirichlet integral.

The purpose of this paper is to study the NED-sets in higher dimensions. We establish for a closed $E \subset \bar{R}^{n}$ the following measure-theoretic conditions:

(a) If $E$ is NED, then the $n$-dimensional Lebesgue measure of $E$ is zero.

(b) If the $n$-1-dimensional Hausdorff measure of $E$ is zero, then $E$ is NED.

In addition we prove the topological condition

(c) If $E$ is NED, then $\operatorname{dim} E \leqq n-2$.

For $n=2$, these are well-known properties of the $O_{A D}$-sets.

2. Terminology. The points of $R^{n}$ are treated as vectors. We denote by $B^{n}$ the $n$-ball $|x|<1$, where $|x|$ is the norm of the vector $x$. If $x \in R^{n}$, $A \subset R^{n}, C \subset R^{n}$ and $r$ is a real number, we let

$$
\begin{aligned}
A+x & =\{a+x: a \in A\}, \\
A \pm C & =\{a \pm c: a \in A, c \in C\}, \\
r A & =\{r a: a \in A\} .
\end{aligned}
$$

1) For the definition of the module, see Section 2 . 
With this notation, we have for instance

$$
A+r B^{n}=\{x: d(x, A)<r\},
$$

where $d$ is the Euclidean distance. The complement of $A$ with respect to $C$ is denoted by $C \sim A$.

For $A \subset R^{n}$, we let $m_{n}(A)$ be its $n$-dimensional Lebesgue outer measure. Put $\Omega_{n}=m_{n}\left(B^{n}\right)$. The $p$-dimensional $\left.{ }^{2}\right)$ Hausdorff outer measure $m_{p}(A)$ of $A$ is defined as follows: Let $\varepsilon>0$ and let $B_{1}, B_{2}, \ldots$ be a countable covering of $A$ by open $n$-balls with radii $r_{1}, r_{2}, \ldots$ such that $r_{i}<\varepsilon$. Set

$$
m_{p}^{\varepsilon}(A)=\inf \sum_{i=1}^{\infty} \Omega_{p} r_{i}^{p}
$$

over all such coverings. Then

$$
m_{p}(A)=\lim _{\varepsilon \rightarrow 0+} m_{p}^{\varepsilon}(A)=\sup _{\varepsilon>0} m_{p}^{\varepsilon}(A) .
$$

The measure of a set $A$ which contains the point at infinity is defined as the measure of $A \sim\{\infty\}$.

Let $\Gamma$ be a farnily of curves in $\bar{R}^{n}$. We define $F(\Gamma)$ as the family of all non-negative Borel-measurable (= Baire) functions, defined in $R^{n}$ and satisfying the condition.

$$
\int_{\gamma} \varrho d s \geqq 1
$$

for every $\gamma \in \Gamma$. The greatest lower bound

$$
M(\Gamma)=\inf _{\varrho \in F(\Gamma)} \int_{R^{n}} \varrho^{n} d \tau
$$

is the module of $\Gamma$. Here $d \tau$ is the $n$-dimensional volume element. We will usually omit the domain of integration if it is the whole $R^{n}$. For the properties of the module of a curve family, see [8].

In this paper, we will only consider curve families of the following type. Let $G$ be an open set in $\bar{R}^{n}$ and let $F_{1}, F_{2}$ be two disjoint continua in $G$. Then $I$ is the family of all rectifiable ${ }^{3}$ ) arcs which join $F_{1}$ and $F_{2}$ in $G$. We say that $\Gamma$ is the family joining $F_{1}$ and $F_{2}$ in $G$. The number $1 / M(\Gamma)$ is called the extremal distance between $F_{1}$ and $F_{2}$ in $G$.

3. We first state some general remarks on NED-sets. It is clear that a closed subset of an NED-set is also NED. Furthermore, since the module

2) We shall consider only the case $p=n-1$.

3) This restriction is unessential, because the non-rectifiable curves have no influence on the module of a curve family. See [8], p. 8. 
of a curve family is a conformal invariant, the image of an NED-set under a conformal mapping of $\bar{R}^{n}$ is also NED. If $E$ disconnects $\bar{R}^{n}$, it cannot be NED. For, then we can choose two non-degenerate continua $F_{1}, F_{2}$ from different components of $\bar{R}^{n} \sim E$. The $\left.M(\Gamma)>0^{4}\right)$ while $M\left(\Gamma_{E}\right)=$ $M(\varnothing)=0$.

4. We next prove the proposition (a) mentioned in the introduction.

Theorem 1. If $E \subset \bar{R}^{n}$ is NED, then $m_{n}(E)=0$.

Proof. Choose two distinct points $a, b$ from the complement of $E$ and map $\bar{R}^{n}$ conformally onto itself so that $a, b$ are mapped into $0, \infty$, respectively. The image $E^{\prime}$ of $E$ is still NED. Since $E^{\prime}$ is closed, we can find positive numbers $r_{1}, r_{2}$ such that $E^{\prime}$ is contained in the spherical ring

$$
A=\left\{x: r_{1}<|x|<r_{2}\right\} \text {. }
$$

Let $F_{1}, F_{2}$ be the components of $\bar{R}^{n} \sim A$. If $\Gamma$ is the family joining $F_{1}$ and $F_{2}$, we have

$$
M(T)=\int \varrho^{n} d \tau,
$$

where $\varrho \in F(T)$ is defined by

$$
\begin{array}{ll}
\varrho(x)=\frac{1}{|x| \log \frac{r_{2}}{r_{1}}} \text { for } x \in A, \\
\varrho(x)=0 \quad \text { for } x \in R^{n} \sim A,
\end{array}
$$

(see [8], p. 8). We define a function $\varrho_{1}$ by

$$
\begin{array}{ll}
\varrho_{1}(x)=\varrho(x) & \text { for } \quad x \in R^{n} \sim E^{\prime}, \\
\varrho_{1}(x)==0 & \text { for } \quad x \in E^{\prime} .
\end{array}
$$

Then $\varrho_{1} \in F\left(\Gamma_{E^{\prime}}\right)$, whence

$$
M\left(\Gamma_{E^{\prime}}\right) \leqq \int \varrho_{1}^{n} d \tau \leqq \int \varrho^{n} d \tau=M(\Gamma) .
$$

Because $E^{\prime}$ is NED, we have $M\left(\Gamma_{E^{\prime}}\right)=M(\Gamma)$. Thus

$$
0=\int_{R^{n}}\left(\varrho^{n}-\varrho_{1}^{n}\right) d \tau=\int_{E^{\prime}} \varrho^{n} d \tau .
$$

Since $\varrho(x)>0$ for $x \in E^{\prime}$, this implies $m_{n}\left(E^{\prime}\right)=0$. Hence, $m_{n}(E)==0$, q.e.d.

4) See Loewner [6]. 
5. In order to prove the proposition (b) in the introduction we need five lemmas.

Lemma 1. Let $A \subset R^{n}$ be the spherical ring $r_{1}<|x|<r_{2}$ and let $F_{1}, F_{2}$ be two disjoint subsets of $A$ such that every sphere $|x|=r$, $r_{1}<r<r_{2}$, meets both $F_{1}$ and $F_{2}$. If $\Gamma$ is the family joining $F_{1}$ and $F_{2}$ in $A$, then

$$
M(\Gamma) \geqq c_{n} \log \frac{r_{2}}{r_{1}},
$$

where $c_{n}$ is a constant depending only on $n$.

In the case $n=3$, this was proved in [8] (Theorem 3.9), with $c_{3}=$ $1 / 200$. The general case can be proved in an analogous manner.

6. Let $G$ be a domain in $\bar{R}^{n}$ and let $F_{1}, F_{2}$ be two disjoint nondegenerate bounded continua in $G$. Denote by $\Gamma$ the family which joins $F_{1}$ and $F_{2}$ in $G$. Let $\delta$ be the smallest of the numbers $d\left(F_{1}, \bar{R}^{n} \sim G\right)$, $d\left(F_{2}, \bar{R}^{n} \sim G\right)$ and $\frac{1}{2} d\left(F_{1}, F_{2}\right)$. For each $0<r<\delta$ denote

$$
F_{i}^{r}=F_{i}+r \bar{B}^{n}
$$

$i=1,2$. Furthermore, let $\Gamma^{r}$ be the family joining $F_{1}^{r}$ and $F_{2}^{r}$ in $G$. For each $\varrho \in F(T)$ put

$$
L(r, \varrho)=\inf _{\gamma \in \Gamma^{r}} \int_{\gamma} \varrho d s .
$$

As $r$ decreases, $L(r, \varrho)$ increases. Thus the $\operatorname{limit} \lim L(r, \varrho)$ exists.

Lemma 2. If $\varrho \in F(T)$ and $\varrho$ is $L^{n}$-integrable over $\stackrel{r \rightarrow 0+}{R^{n}}$, then $\left.\lim L(r, \varrho) \geqq 1^{5}\right)$.

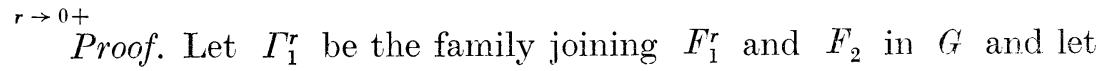

$$
L_{1}(r, \varrho)=\inf _{\gamma \in \Gamma_{i}^{r}} \int_{\gamma} \varrho d s .
$$

We first prove that $\lim L_{1}(r, \varrho) \geqq 1$.

Suppose $\lim _{r \rightarrow 0+} L_{1}(r, \varrho)<q<1$. Set $R=\min \left(\delta, \frac{1}{2} d\left(F_{1}\right)\right)$ and let $0<r<R$. Then there exists an arc $\gamma$ in $\Gamma_{1}^{r}$ such that

$$
\int_{\gamma} \varrho d s<q .
$$

Let $a$ be the endpoint of $\gamma$ which belongs to $F_{1}^{r}$. Then there exists a point $b$ in $F_{1}$ such that $|a-b| \leqq r$. For each $s$ such that $r<s<R$,

$\left.{ }^{5}\right)$ A similar lemma is established in a recent paper [5] of Gehring. 
the sphere $|x-b|=s$ meets both $F_{1}$ and $\gamma$. Let $\Gamma_{r}^{\prime}$ be the family joining $F_{1}$ and $\gamma$ in $G$. Because the ring $r<|x-b|<R$ lies in $G$, Lemma 1 implies

$$
M\left(\Gamma_{r}^{\prime}\right) \geqq c_{n} \log \frac{R}{r}
$$

Let $\gamma^{\prime} \in \Gamma_{r}^{\prime}$. Because the continuum $\gamma \cup \gamma^{\prime}$ joins $F_{1}$ and $F_{2}$ in $G$, there exists an arc $\gamma^{\prime \prime}$ in $\Gamma$ such that $\gamma^{\prime \prime} \subset \gamma \cup \gamma^{\prime}$. Thus,

$$
1 \leqq \int_{\gamma^{\prime \prime}} \varrho d s \leqq \int_{\gamma} \varrho d s+\int_{\gamma^{\prime}} \varrho d s<q+\int_{\gamma^{\prime}} \varrho d s
$$

for each $\gamma^{\prime} \in \Gamma_{r}^{\prime}$. Hence, the function $\varrho /(1-q)$ belongs to $F^{\prime}\left(I_{r}^{\prime}\right)$ so that

$$
M\left(\Gamma_{r}^{\prime}\right) \leqq \frac{1}{(1-q)^{n}} \int \varrho^{n} d \tau
$$

Together with (2) this yields

$$
(1-q)^{n} c_{n} \log \frac{R}{r} \leqq \int \varrho^{n} d \tau .
$$

Finally, if we let $r \rightarrow 0$, we obtain a contradiction. Thus $\lim _{r \rightarrow 0+} L_{1}(r, \varrho) \geqq 1$.

Now let $0<\varepsilon<1$. By the above, there exists a positive number $r_{1}$ such that

$$
L_{1}\left(r_{1}, \varrho\right)>1-\varepsilon
$$

We apply the first part of the above proof replacing $F_{1}$ by $F_{2}, F_{2}$ by $F_{1}^{r_{1}}$ and $\varrho$ by $\varrho /(1-\varepsilon)$. We thus find an $r_{2}>0$ such that

$$
\int_{\gamma} \frac{\varrho}{1-\varepsilon} d s>1-\varepsilon
$$

for each $\gamma$ joining $F_{1}^{r_{1}}$ and $F_{2}^{r_{2}}$. Thus,

$$
\int_{\gamma} \varrho d s>(1-\varepsilon)^{2}
$$

whenever $\gamma \in \Gamma^{r}$ and $r<\min \left(r_{1}, r_{2}\right)$. This completes the proof of the lemma.

7. Next we require some estimates for the measure of sets $E+\gamma$ where $\gamma$ is a rectifiable arc. 
Lemma 3. Let $\gamma \subset R^{n}$ be a rectifiable arc of length $l$ and let $r>0$. Then

$$
m_{n}\left(\gamma+r B^{n}\right) \leqq r^{n-1}\left(\Omega_{n} r+\Omega_{n-1} l\right) .
$$

If $\gamma$ is a segment of line, (3) holds with equality.

For $n=2$, this is proved in Apostol [2], p. 285. The proof for the general case is similar.

Lemma 4. Let $\gamma \subset R^{n}$ be a rectifiable arc of length $l$ and let $E$ be any subset of $R^{n}$. Then

$$
m_{n}(E+\gamma) \leqq l m_{n-1}(E)
$$

The bound is sharp.

Proof. If $m_{n-1}(E)=\infty$, the lemma is trivial. Assume $m_{n-1}(E)$ is finite. Let $\varepsilon>0$. Cover $E$ with balls $B_{1}, B_{2}, \ldots$ such that their radii $r_{i}<\varepsilon$ and

$$
\sum_{i=1}^{\infty} \Omega_{n-1} r_{i}^{n-1}<m_{n-1}(E)+\varepsilon .
$$

Then

$$
E+\gamma \subset \bigcup_{i=1}^{\infty}\left(B_{i}+\gamma\right)
$$

Hence,

$$
m_{n}(E+\gamma) \leqq \sum_{i=1}^{\infty} m_{n}\left(B_{i}+\gamma\right) .
$$

By Lemma 3,

$$
m_{n}\left(B_{i}+\gamma\right) \leqq r_{i}^{n-1}\left(\Omega_{n} r_{i}+\Omega_{n-1} l\right)
$$

Thus,

$$
\begin{aligned}
m_{n}(E+\gamma) & \leqq\left(\Omega_{n} \varepsilon+\Omega_{n-1} l\right) \sum_{i=1}^{\infty} r_{i}^{n-1} \\
& \leqq\left(\Omega_{n} \varepsilon+\Omega_{n-1} l\right) \frac{m_{n-1}(E)+\varepsilon}{\Omega_{n-1}}
\end{aligned}
$$

As $\varepsilon \rightarrow 0$, this gives (4).

If $E$ is contained in an $n$-1-dimensional linear subspace $T$ of $R^{n}$ and if $\gamma$ is a line segment perpendicular to $T$, then (4) holds with equality.

Lemma 5. Let $\gamma$ be a rectifiable arc in $R^{n}$ and let $E \subset R^{n}$ such that $m_{n-1}(E)=0$. Then

$$
(\gamma+x) \cap E=\varnothing
$$

or almost every $x \in R^{n}$. 
Proof. Obviously,

$$
\{x:(\gamma+x) \cap E \neq \varnothing\}=E-\gamma .
$$

Because $-\gamma$ is rectifiable, Lemma 4 implies that $m_{n}(E-\gamma)=0$, q.e.d.

8. We are now ready to prove our main theorem.

Theorem 2. Let $E$ be a closed subset of $\bar{R}^{n}$ such that $m_{n-1}(E)=0$. Then $E$ is NED.

Proof. Let $F_{1}, F_{2}$ be disjoint continua in $\bar{R}^{n} \sim E$ and let $T$ be the family joining $F_{1}$ and $F_{2}$ in $\bar{R}^{n}$. We must prove that

$$
M(\Gamma) \leqq M\left(\Gamma_{E}\right)
$$

where, as before, $\Gamma_{E}$ is the subfamily of $\Gamma$ whose members do not meet $E$.

Performing a preliminary conformal mapping, we may assume that $F_{1}, F_{2}$ are bounded. We may also assume that $F_{1}, F_{2}$ are non-degenerate, because otherwise $M(\Gamma)=0$ and $(5)$ holds trivially. Let $0<\varepsilon<1$. Choose a function $\varrho \in F\left(\Gamma_{E}\right)$ such that

$$
\int \varrho^{n} d \tau<M\left(\Gamma_{E}\right)+\varepsilon .
$$

By Lemma 2 there exists a positive number $r$ such that

$$
L(r, \varrho)>1-\varepsilon .
$$

Here

$$
L(r, \varrho)=\inf \int_{\gamma} \varrho d s,
$$

where the infimum is taken over all rectifiable ares $\gamma$ which join $F_{1}+r \bar{B}^{n}$ and $F_{2}+r \bar{B}^{n}$ in $\bar{R}^{n} \sim E$.

We construct the spherical $r$-average function $\varrho_{1}$ of $\varrho$,

$$
\varrho_{1}(x)=\frac{1}{\Omega_{n} r^{n}} \int_{\mid y_{i}<r} \varrho(x+y) d \tau .
$$

We next prove that $\varrho_{1} /(1-\varepsilon)$ belongs to $F(T)$.

Let $\gamma \in \Gamma$ and let $f:[0, l] \rightarrow R^{n}$ be the representation of $\gamma$ parametrized with respect to arc-length. Then

$$
\int_{\gamma} \varrho_{1} d s=\frac{1}{\Omega_{n} r^{n}} \int_{0}^{l}\left(\int_{|y|<r} \varrho(f(s)+y) d \tau\right) d s .
$$


The function $\varrho(f(s)+y)$ is Borel-measurable in $R^{1} \times R^{n}$. By Fubini's theorem, we may interchange the order of integration. Thus

$$
\int_{\gamma} \varrho_{1} d s=\frac{1}{\Omega_{n} r^{n}} \int_{|y|<r}\left(\int_{\gamma+y} \varrho d s\right) d \cdot \tau .
$$

The are $\gamma+y$ joins $F_{1}+r \bar{B}^{n}$ and $F_{2}+r \bar{B}^{n}$ for every $|y|<r$. By Lemma $5,(\gamma+y) \cap E=\varnothing$ for almost every $y$. Hence,

$$
\int_{\gamma+y} \varrho d s \geqq L(r, \varrho)>1-\varepsilon
$$

for almost all $y,|y|<r$. Consequently, (7) yields

$$
\int_{\gamma} \varrho_{1} d s \geqq 1-\varepsilon .
$$

This proves that $\varrho_{1} /(1-\varepsilon) \in F(\Gamma)$. We thus have the estimate

$$
M(\Gamma) \leqq \frac{1}{(1-\varepsilon)^{n}} \int \varrho_{1}^{n} d \tau \text {. }
$$

An application of Hölder's inequality gives

$$
\int \varrho_{1}^{n} d \tau \leqq \int \varrho^{n} d \tau
$$

(cf. Morrey [7], p. 687). Combining (6), (8) and (9) we obtain

$$
M(I) \leqq \frac{M\left(\Gamma_{E}\right)+\varepsilon}{(1-\varepsilon)^{n}},
$$

and letting $\varepsilon \rightarrow 0$ yields (8).

Remark. The condition $m_{n-1}(E)=0$ of the theorem cannot be replaced by $m_{n-1}(E)<\infty$. For instance, the $n-1$-sphere $|x|=1$ has finite $n-1$ measure, but because it disconnects $K^{n}$, it cannot be NED.

9. We next prove the topological condition (c).

Theorem 3. If $E \subset \bar{R}^{n}$ is $\mathrm{NED}$, then $\operatorname{dim} E \leqq n-2$.

Proof. If $\operatorname{dim} E=n$, then $E$ contains an inner point. Thus $m_{n}(E)$ $>0$, which is impossible by Theorem 1 .

Assume that $\operatorname{dim} E=: n-1$. 'Then, by results due to Frankl and Pontrjagin $[3,4]$, there exists a domain $G$ in $\bar{R}^{n}$ such that $G \sim E$ is not connected. Let $a \in G \cap E$ be a common boundary point of two components $U$ and $V$ of $G \sim E$. We may assume that $a \neq \infty$. Fix $R>0$ such that the ball $a+2 R B^{n}$ is contained in $G$. Let $0<r<R$. 
Choose points $x$ and $y$ in $U \cap\left(a+r B^{n}\right)$ and $V \cap\left(a+r B^{n}\right)$, respectively. Because $\bar{R}^{n} \sim E$ is connected (see Section 3), there is an arc $\gamma$ which joins $x$ and $y$ in $\bar{R}^{n} \sim E$. Let $\alpha \subset a+R \bar{B}^{n}$ be the subarc of $\gamma$ which joins $x$ to the boundary sphere of $a+R \bar{B}^{n}$, and let $\beta$ be the corresponding are for $y$. Consider the family $\Gamma$ which joins $\alpha$ and $\beta$. By Lemma 1,

$$
M(\Gamma) \geqq c_{n} \log \frac{R}{\gamma} .
$$

Next define a function $\varrho$ by

$$
\begin{aligned}
& \varrho(x)=\frac{1}{2 R} \text { for } x \in a+2 R B^{n}, \\
& \varrho(x)=0 \text { otherwise. }
\end{aligned}
$$

Obviously, $\varrho \in F\left(\Gamma_{E}\right)$. Consequently,

$$
M\left(\Gamma_{E}\right) \leqq \int \varrho^{n} d \tau=\Omega_{n}
$$

Because $E$ is NED, we have $M(I)==M\left(I_{E}\right)$. Hence, (10) and (11) yield

$$
c_{n} \log \frac{R}{r} \leqq \Omega_{n}
$$

Letting $r \rightarrow 0$ gives the desired contradiction.

Remark. Theorem 3 has the following consequence: Let $E$ be a closed subset of $R^{n-1}$ such that $E$ contains an inner point. Then no topological imbedding of $E$ into $\bar{R}^{n}$ is NED. In particular, if we consider $R^{n-1}$ as a subset of $R^{n}, E$ is not NED with respect to $\bar{R}^{n}$. 


\section{References}

[1] L. V. Ahlfors - A. Beurling: Conformal invariants and function-theoretic null-sets. - Acta Math. 83, 1950, 101-129.

[2] T. M. Apostol: Mathematical analysis. - Addison-Wesley, 1957.

[3] F. FrankL: Charakterisierung der $n$-l-dimensionalen abgeschlossenen Mengen des $R^{n}$. - Math. Ann. 103, 1930, 784-787.

[4] F. Frankl - L. Pontruagin: Ein Knotensatz mit Anwendung auf die Dimensionstheorie. - Math. Ann. 102, 1930, 785- 789.

[5] F. W. Gehring: Extremal length definitions for the conformal capacity of rings in space. - Mich. Math. J. 9, 1962, $137-150$.

[6] C. Loewner: On the conformal capacity in space. - J. Math. Mech. 8, 1959, $411-414$.

[7] C. B. Morrey: A class of representations of manifolds. - Amer. J. Math. 55, $1933,683-707$.

[8] J. VäIs̈̈LÄ: On quasiconformal mappings in space. - Ann. Acad. Sci. Fenn. A I 298, 1961, 1-36.

University of Helsinki 